\section{RMD Open}

Rheumatic \&

Musculoskeletal Diseases

\title{
Long-term efficacy and safety of antitumour necrosis factor alpha treatment in rhupus: an open-label study of 15 patients
}

François Danion, ${ }^{1,2}$ Laetitia Sparsa, ${ }^{3}$ Laurent Arnaud, ${ }^{1,2}$ Ghada Alsaleh, ${ }^{4}$ François Lefebvre, ${ }^{5}$ Vincent Gies, ${ }^{6}$ Thierry Martin, ${ }^{6,7}$ Cédric Lukas, ${ }^{8}$ Jean Durckel, ${ }^{9}$ Marc Ardizzone, ${ }^{3}$ Rose-Marie Javier, ${ }^{1,2}$ Jean-François Kleinmann, ${ }^{1,2}$ Paul Moreau, ${ }^{10}$ Gilles Blaison, ${ }^{11}$ Joelle Goetz, ${ }^{12}$ Emmanuel Chatelus, ${ }^{1,2}$ Jacques-Eric Gottenberg, ${ }^{1,2,6}$ Jean Sibilia, ${ }^{1,2,4}$ Christelle Sordet ${ }^{1,2}$

To cite: Danion F, Sparsa L, Arnaud L, et al. Long-term efficacy and safety of antitumour necrosis factor alpha treatment in rhupus: an open-label study of 15 patients. RMD Open 2017;3:e000555. doi:10.1136/ rmdopen-2017-000555

\section{- Prepublication history for this paper is available online. To view these files, please visit the journal online (http://dx.doi. org/10.1136/rmdopen-2017- 000555).}

JS and CS contributed equally, FD and LS contributed equally.

Received 6 August 2017 Revised 4 October 2017 Accepted 21 October 2017

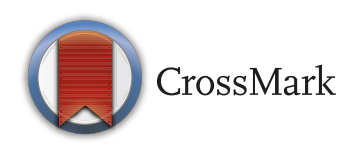

For numbered affiliations see end of article.

Correspondence to Dr Christelle Sordet; Christelle.SORDET@chrustrasbourg.fr

\section{ABSTRACT}

Background The efficacy of antitumour necrosis factor alpha (anti-TNF- $\alpha$ ) treatment is well recognised in rheumatoid arthritis (RA) but remains controversial in systemic lupus erythematosus (SLE). Therefore, the role of anti-TNF- $\alpha$ treatment in 'Rhupus', a disease sharing features of RA and SLE, is still debated.

Objective To evaluate the efficacy and tolerance of antiTNF- $\alpha$ in patients with rhupus.

Methods Fifteen patients with rhupus with Disease Activity Score 28 (DAS 28) >3.2 despite conventional disease-modifying anti-rheumatic drugs were included in an open-label study. Patients were monitored at months (M) 3, 6, 12, 24 and 60 with SLE Disease Activity Index (SLEDAI) and DAS 28. Statistical analyses were performed using Bayesian methods and Prob $>97.5 \%$ was considered significant.

Results Twelve patients were treated with etanercept for a median duration of 62.5 (range: 6-112) months and three patients by adalimumab during 36.0 (range: 4-52) months. At baseline, median DAS 28 and SLEDAl were 5.94 (4.83-8.09) and 6 (4-8), respectively. DAS 28 and SLEDAI decreased significantly after 3 months, respectively, to $3.70(1.80-6.42)$ and 4 (0-6) (Prob $>99.9 \%$, for both). These changes persisted at M6, M12, M24 and M60 (Prob >99.9\%, for all). Median prednisone dose decreased significantly from $15(5-35) \mathrm{mg} /$ day to 5 (0-20) $\mathrm{mg} /$ day after 6 months and over the follow-up (Prob $>99.9 \%$, for all). Tolerance was acceptable, with a severe infection rate of 3.0 per 100 patient-years.

Conclusion This pilot study suggests that anti-TNF- $\alpha$ is effective in patients with rhupus with refractive arthritis and has an acceptable safety profile.

\section{INTRODUCTION}

Rhupus is a rare association, defined by the coexistence in the same patient of both systemic lupus erythematosus (SLE) and rheumatoid arthritis (RA). In 1971, Schur ${ }^{1}$ used the term 'rhupus' to describe patients

\section{Key messages}

What is already known about this subject?

- Only few biologics have been evaluated for rhupus, a rare association of systemic lupus erythematosus (SLE) and rheumatoid arthritis, when synthetic disease-modifying anti-rheumatic drugs were non-effective.

What does this study add?

- This study showed a good efficacy of anti-tumour necrosis factor alpha in decreasing Disease Activity Score 28, SLE Disease Activity Index and glucocorticoid dosage in 15 patients with rhupus, with an acceptable safety profile and no lupus flare.

How might this impact on clinical practice?

- This pilot study gives an alternative therapy to rituximab in patients with refractive rhupus especially in which arthritis is the most prominent component, but needs comparative studies to confirm formally the efficacy and the tolerance.

satisfying the criteria for both SLE and RA, without establishing whether this coexistence was a distinct immunoclinical entity or only the coincident presence of both conditions in the same patient.

Tumour necrosis factor- $\alpha$ (TNF- $\alpha$ ) plays an important role in propagating inflammatory diseases like RA and SLE. If the good efficacy and safety of anti-TNF- $\alpha$ treatments have been recognised for more than 20 years in $\mathrm{RA}$, their role in SLE treatment remains very controversial. ${ }^{2}$ Anti-TNF- $\alpha$ therapy often induces antinuclear antibodies, and cases of drug-induced SLE have been reported in patients with RA following anti-TNF- $\alpha$ therapy. ${ }^{3-5}$ The number of patients treated 
with infliximab who developed antinuclear antibodies and antibodies against double-stranded DNA (dsDNA) of IgG class increased significantly, respectively, from $24 \%$ to $77 \%$ and from $2 \%$ to $67 \%$ after 30 weeks of treatment in a study of 53 patients with RA. ${ }^{3}$ In some animal models, treatment with TNF- $\alpha$ delays the progression of lupus nephritis. ${ }^{67}$ On the contrary, an increased expression of TNF- $\alpha$ is detectable in renal biopsies of active lupus nephritis, as well as in refractory skin lesions from patients with subacute cutaneous lupus. ${ }^{8}{ }^{9}$ Also, TNF- $\alpha$ induces damage on keratinocytes, which results in Ro autoantigen release. ${ }^{10}$

Blocking TNF- $\alpha$ could therefore be beneficial in selected patients with SLE with renal, skin or articular involvement. Currently, the experience of antiTNF- $\alpha$ in SLE is limited to small open-label trials and case reports, the majority using infliximab. Aringer et $a l$ have shown that short-term induction therapy with anti-TNF- $\alpha$ induced long-term improvement in six of nine patients with renal nephritis. ${ }^{11-13}$ However, the treatment also increased autoantibodies to nuclear antigens and phospholipids. Also, decrease in SLE Disease Activity Index (SLEDAI) and glucocorticoid sparing effect were observed in patients treated with infliximab in a study including 27 patients with SLE. ${ }^{14}$ Another study also showed clinical improvement in 38/42 patients with SLE with cardiopulmonary and/ or articular involvement. ${ }^{15}$ Nevertheless, for a majority of teams, anti-TNF- $\alpha$ treatments should not be used in routine clinical practice in SLE.

To date, there are no studies regarding the use of anti-TNF- $\alpha$ in patients with rhupus. Very limited evidence is available regarding the efficacy of rituximab in lupus arthritis cases, including four open-label of rhupus series of less than 10 patients each, ${ }^{16-19}$ and a meta-analysis. ${ }^{20}$ Abatacept showed also some efficacy in the treatment of three and six patients with rhupus. ${ }^{19}{ }^{21}$ The post-hoc analysis of the negative phase III study of abatacept in SLE showed a significant effect in the subgroup of patients presenting with polysynovitis as the main manifestation. ${ }^{22}$

In this open-label study, we analysed the long-term efficacy and the safety profile of anti-TNF- $\alpha$ treatment in 15 patients with rhupus.

\section{PATIENTS AND METHODS \\ Study design}

We conducted an open, single-centre, open-label study from 2003 to September 2012. Patients were included if they had been diagnosed with (1) rhupus, defined as patients fulfilling the American College of Rheumatology (ACR) criteria for both SLE (1997) and RA (1987) ${ }^{23}$ 24; and had (2) an active disease, defined by Disease Activity Score 28 (DAS 28) >3.2 despite conventional synthetic disease-modifying anti-rheumatic drugs (DMARDs). Patients with active tuberculosis, hepatitis C or B virus, HIV, history of lymphoproliferative syndrome, history of malignant tumour, congestive heart failure (New York Heart Association class III or IV) and patients who had history of central nervous impairment were excluded. Informed consent was obtained from all patients.

\section{Follow-up}

After anti-TNF- $\alpha$ initiation, patients were monitored at months 3 (M3), 6 (M6), 12 (M12), 24 (M24) and 60 (M60). At each visit, clinical examination, biological assessment, and an evaluation of the efficacy and the safety of the treatment were performed. SLE and RA disease activities were assessed using Safety of Estrogens in Lupus Erythematosus National Assessment-SLEDAI (SELENA-SLEDAI) and DAS $28 \mathrm{C}$ reactive protein. ${ }^{25} 26$ Response to anti-TNF- $\alpha$ treatment was assessed by the DAS 28, European League Against Rheumatism (EULAR) response,${ }^{27}$ and changes in the SLEDAI and in the glucocorticoid dose. ${ }^{1428}$

Severe infections, cardiovascular events, neoplasia and lupus flares were recorded. Lupus flares were defined by a new renal, neuropsychiatric or cardiorespiratory involvement or an increase of $\geq 3$ points in SLEDAI. ${ }^{25}$ Serious infections were defined by an infection requiring hospitalisation and/or intravenous antibiotic therapy or death attributable to the infection. Cardiovascular events were defined as acute coronary syndrome, symptomatic peripheral vascular disease, stroke and transient ischaemic attack.

\section{Statistical analysis}

Descriptive statistics used the median (range) for quantitative variables and the number (percentage) for qualitative ones.

Statistical analyses were performed using Bayesian methods. Beta mixed regressions were used to estimate the evolution of DAS 28, SLEDAI and glucocorticoid dose. The correlation between patient values was handled through an unstructured covariance matrix of random effects.

Parameters 95\% credibility intervals and posterior probabilities (Prob) were calculated. Non-informative priors were used. A result was considered significant when the posterior probability that there is a difference between two values was more than $97.5 \%$ (Prob >97.5\%) or less than $2.5 \%$ (Prob $<2.5 \%$ ). The statistical analyses were performed using WinBUGS V.1.4 software.

\section{RESULTS}

\section{Patients' characteristics}

Fifteen patients were included (14 female). The median age was 52 years (range: 20-79). The median follow-up time before anti-TNF- $\alpha$ initiation was 12 years (range: 4-35). The median number of ACR criteria for RA was 6 (range: 4-7) and 5 (range: 4-6) for SLE. The detailed characteristics of the 15 patients treated by anti-TNF- $\alpha$ are shown in table 1 . 
Table 1 Clinicobiological and radiological characteristics of patients with rhupus

\begin{tabular}{|c|c|c|}
\hline & & $n=15$ \\
\hline \multicolumn{2}{|c|}{ Age (years), median (range) } & $52(20-79)$ \\
\hline \multicolumn{2}{|l|}{ Sex (female/male) } & $14 / 1$ \\
\hline \multicolumn{2}{|c|}{ Age at onset of disease (years), median (range) } & $36(11-64)$ \\
\hline \multirow[t]{3}{*}{ Onset disease, n (\%) } & RA & $6(40 \%)$ \\
\hline & SLE & $7(47 \%)$ \\
\hline & Rhupus & $2(13 \%)$ \\
\hline \multicolumn{2}{|c|}{$\begin{array}{l}\text { Interval between first symptom and rhupus } \\
\text { diagnosis (years), median }\end{array}$} & $3(0-29)$ \\
\hline
\end{tabular}

SLE criteria

\begin{tabular}{|c|c|}
\hline 1997 ACR criteria, median (range) & $5(4-6)$ \\
\hline Malar rash, $\mathrm{n}(\%)$ & $8(53 \%)$ \\
\hline Discoid lupus, $n(\%)$ & $2(13 \%)$ \\
\hline Photosensibility, n (\%) & $14(93 \%)$ \\
\hline Arthritis, n (\%) & $15(100 \%)$ \\
\hline Pericarditis or pleuritis, $\mathrm{n}(\%)$ & $2(13 \%)$ \\
\hline Renal, $n(\%)$ & $1(7 \%)$ \\
\hline Neurological disorder, n (\%) & 0 \\
\hline Haematological, n (\%) & $13(87 \%)$ \\
\hline \multicolumn{2}{|l|}{ RA criteria } \\
\hline ACR criteria, median (range) & $6(4-7)$ \\
\hline Morning stiffness (>60 min), n (\%) & $15(100 \%)$ \\
\hline Arthritis > 3 joints, $n(\%)$ & $15(100 \%)$ \\
\hline Arthritis of hands, $\mathrm{n}(\%)$ & $15(100 \%)$ \\
\hline Symmetrical arthritis, $\mathrm{n}(\%)$ & $15(100 \%)$ \\
\hline Rheumatoid nodules, n (\%) & $6(40 \%)$ \\
\hline $\begin{array}{l}\text { RF, } n(\%) \\
\quad \text { Median (range), }{ }^{*}(\mathrm{IU} / \mathrm{mL})\end{array}$ & $\begin{array}{l}10(67 \%) \\
57.5(17-244)\end{array}$ \\
\hline X-ray changes (erosions), n (\%) & $13(87 \%)$ \\
\hline \multicolumn{2}{|l|}{ Antibodies } \\
\hline $\begin{array}{l}\text { Anti-CCP, n (\%) } \\
\text { Median (range), }{ }^{*}(\mathrm{IU} / \mathrm{mL})\end{array}$ & $\begin{array}{l}9(60 \%) \\
540(167-1240)\end{array}$ \\
\hline Positive ANA, n (\%) & $15(100 \%)$ \\
\hline $\begin{array}{l}\text { Anti-dsDNA, n (\%) } \\
\text { Median (range), }{ }^{*}(\mathrm{IU} / \mathrm{mL})\end{array}$ & $\begin{array}{l}10(67 \%) \\
99.5(51-289)\end{array}$ \\
\hline Anti-Ro/SSA, n (\%) & $3(20 \%)$ \\
\hline Anti-La/SSB, n (\%) & $1(7 \%)$ \\
\hline Anti-Sm, n (\%) & $1(7 \%)$ \\
\hline Anti-RNP, n (\%) & 2 (13\%) \\
\hline
\end{tabular}

*Median rate and range are calculated for patients with detectable autoantibodies.

ACR, American College of Rheumatology; ANA, antinuclear antibodies; anti-dsDNA, anti-double stranded DNA antibodies; anti-RNP, antiribonucleoprotein antibodies; CCP, cyclic citrullinated peptides; $\mathrm{n}$, number of patients; RA, rheumatoid arthritis; RF, rheumatoid factor; SLE, systemic lupus erythematosus; Sm, anti-Smith; SS, Sjögren's syndrome.

Ten patients had significant antibodies to cardiolipin (>20 IgG Phospholipid Unit / IgM Phospholipid Unit) and/or anticoagulant circulating antibody and/or anti$32 \mathrm{GP1}$, but none had antiphospholipid syndrome. Human leukocyte antigen (HLA) DRB1 genotype was DRB1*01 (n=3), DRB1*03 (n=3), DRB1*04 (n=6),
DRB1*07 $\quad(n=4), \quad$ DRB1*09 $(n=2), \quad$ DRB1*11 $\quad(n=2)$, DRB1*12 $(\mathrm{n}=1), \mathrm{DRB} 1 * 13(\mathrm{n}=2)$ and DRB1*15 $(\mathrm{n}=6)$.

All patients have been treated with methotrexate and 14 with hydroxychloroquine. Patients have also received leflunomide $(n=3)$, azathioprine $(n=2)$, sulfasalazine $(n=1)$ and ciclosporin $(n=1)$ for articular and/or extra-articular manifestations.

At baseline, none of the patients had active renal, neuropsychiatric or cardiorespiratory involvement. Two patients had active mucocutaneous involvements of SLE.

\section{Disease activity and structural damage at baseline}

At baseline, the median DAS 28 was 5.94 (range: 4.838.09 ) and the SLEDAI was 6 (range: $4-8$ ). The median swollen joint count was 9 (range: 4-28), and the median tender joint count was 12 (range: 6-28). The median visual analogue scale evaluated by the patient was $70 / 100$ (range: 45-70). The Sharp/van der Heijde score was available for 13 patients. X-rays of hands and feet showed joint space narrowing in 13/13 patients, and erosions in $11 / 13$. The erosion score was 10 (range: $0-135$ ) and the narrowing/(sub)luxation score was 57 (range: 2-111). The median Sharp/van der Heijde score was 69 (range: 2-246).

\section{Use of treatments}

At baseline 11 patients had methotrexate with a median dose of $15 \mathrm{mg}$ per week (range: 7.5-17.5) and 8 had hydroxychloroquine. The median prednisone dosage was 15 (range: $5-35) \mathrm{mg} /$ day. Eleven patients had a prednisone dosage $\geq 10 \mathrm{mg} /$ day. No other synthetic DMARDs were used.

Twelve patients were treated with etanercept at $50 \mathrm{mg}$ per week and three with adalimumab at $40 \mathrm{mg}$ every 2 weeks.

The median duration of anti-TNF- $\alpha$ treatment was 52 months (range: 4-112), yielding a total treatment exposure of 66.8 patient-years. The median duration of treatment with etanercept was 62.5 months (range: 6-112), that is, 56.1 patient-years, and with adalimumab it was 36.0 months (range: $4-52$ ), that is, 10.7 patient-years.

Anti-TNF- $\alpha$ treatment was discontinued in one patient after 6 months because of primary inefficacy (figure 1). A second patient had a failure of etanercept treatment after 18 months and subsequently responded to adalimumab. None of the patients discontinued anti-TNF- $\alpha$ because of a lupus flare. Two patients who continued with anti-TNF- $\alpha$ treatment were followed up until 43 months (end of the study) and not evaluated at M60.

\section{Response to anti-TNF- $\alpha$ treatment}

Changes in DAS 28 and EULAR response

We observed a significant decrease of DAS 28 at M3, M6, M12, M24 and M60 compared with baseline (Prob >99.9\% for all) (figure 2). According to the EULAR response criteria, all but two patients achieved a good or moderate response at M3 and M6. The detailed proportion of responders at each time-point is shown in figure 2. 


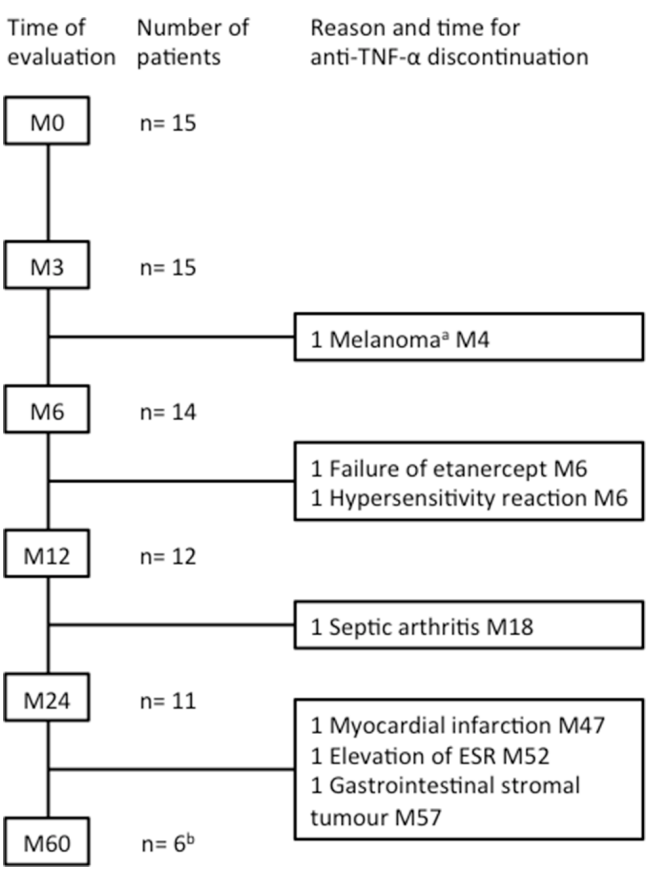

Figure 1 Follow-up and cause of anti-TNF- $\alpha$ discontinuation in 15 patients with rhupus. anti-TNF- $\alpha$, antitumour necrosis factor alpha; ESR, erythrocyte sediment rate; $M$, month; $n$, number of patients. ${ }^{a}$ Exclusion criteria: discovery of an overlooked history of melanoma in the medical history. ${ }^{\mathrm{b}}$ Two patients who continued with antiTNF- $\alpha$ had 43 months of follow-up at the end of the study and were not evaluated at M60.

\section{Changes in SLEDAl}

We observed a significant decrease of SELENA-SLEDAI at M3, M6, M12, M24 and M60 compared with baseline (Prob $>99.9 \%$ for all) (figure 2 ). A decrease of SLEDAI $\geq 4$ was observed in seven patients at M3 and M6, and in nine patients at M12.

A modified SLEDAI (mSLEDAI) was calculated without taking into account the arthritis component of the SLEDAI. This modified score aimed to evaluate the evolution of the SLEDAI independent of the articular component. The median mSLEDAI significantly decreased from 2 at baseline to 0 at M12, M24 and M60 months (Prob $>97.5 \%$, for all).

The two patients with cutaneous manifestations of SLE (malar rash and alopecia) had a clinical improvement.

\section{Changes in associated treatments}

We observed a significant decrease in prednisone dose at M3 (Prob 99.2\%), and at M6, M12, M24 and M60 (Prob $>99.9 \%$ for all) compared with baseline (figure 2 ). Two patients were able to discontinue glucocorticoids completely. The dose of methotrexate was stable for four patients, decreased in three patients, and decreased then increased in one patient. The data were not available for the last three patients. No synthetic DMARDs were introduced during anti-TNF- $\alpha$ treatment.

\section{Tolerance of anti-TNF- $\alpha$ treatment}

Severe infection rate was 3.0 per 100 patient-years. One patient had a septic arthritis of the hip, with required hospitalisation, discontinuation of etanercept and treatment with antibiotics (figure 1). A second patient had a pneumonia at M87 and was treated by ceftriaxone and levofloxacin.

Regarding neoplasia, anti-TNF- $\alpha$ was discontinued in one patient at M4 because of an overlooked history of melanoma. One patient developed a gastrointestinal stromal tumour at M57 and a clear cell renal cell carcinoma 2 years after the discontinuation of anti-TNF- $\alpha$.

Two patients with comorbidities developed cardiovascular events. The first patient (with factor V Leiden mutation, high blood pressure, dyslipidaemia and smoking at 40 pack-years) had a myocardial infarction and symptomatic peripheral vascular disease. The second patient with dyslipidaemia and smoking (40 pack-years) had a myocardial infarction without significant coronary artery disease.

One patient developed a hypersensitivity reaction leading to the discontinuation of etanercept. One patient had a non-lupus rash on the back and on the forearms in the first 3 months of treatment, which disappeared with topical glucocorticoid. One patient had an unexplained elevation of erythrocyte sedimentation rate.

No renal, neuropsychiatric or cardiovascular SLE flare and no increase of SLEDAI $\geq 3$ were observed during the follow-up. No new antibodies to extractable nuclear antigens or anti-dsDNA antibodies were detected during the follow-up. No death occurred.

\section{DISCUSSION}

To the best of our knowledge, this is the first study focusing on the long-term efficacy and tolerance of antiTNF- $\alpha$ treatment in patients with rhupus.

We observed a significant decrease in the DAS 28 and SLEDAI, as well as in the median dose of glucocorticoids. Further, no lupus flares occurred during the follow-up. The tolerance profile was acceptable, with a severe infection rate of 3.0 per 100 patient-years.

Anti-TNF- $\alpha$ treatment resulted in an improvement of articular symptoms with a significant decrease of DAS 28 score. The SLEDAI decreased after 3 months of therapy and this decrease was sustained during the follow-up. Interestingly, this score also decreased without taking into account the arthritis component of the SLEDAI, suggesting that anti-TNF- $\alpha$ also improved non-articular features of lupus in the present study. We also observed a significant decrease in the dose of glucocorticoids.

The main concern regarding the use of anti-TNF- $\alpha$ therapies in SLE has been related to the potential development of autoantibodies. ${ }^{3}{ }^{4}$ In our study, no lupus flare was observed during the long-term follow-up. HLA DR3/4 alleles are associated with high TNF- $\alpha$ response and these haplotypes were observed, respectively, in three and six patients. ${ }^{29}$ The tolerance of anti-TNF- $\alpha$ treatment was acceptable. The rate of serious infection was 3.0 per 100 patient-years, which is close to 2.9 per 100 patient-years over 5 years in the Dutch Rheumatoid 
A

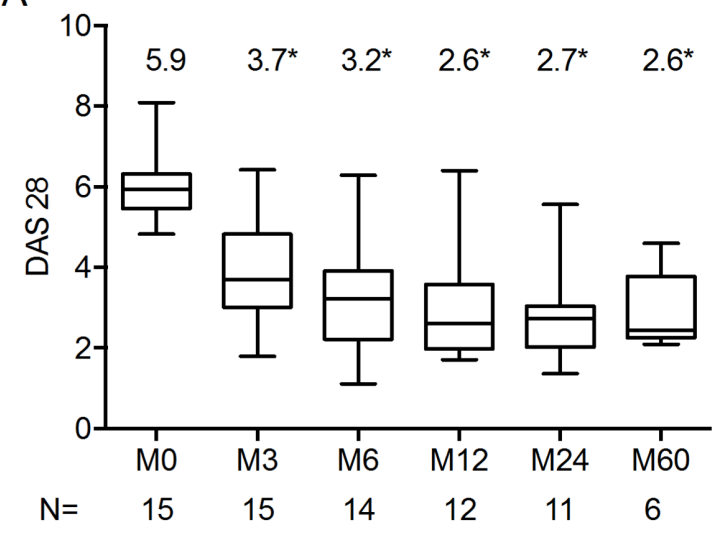

C

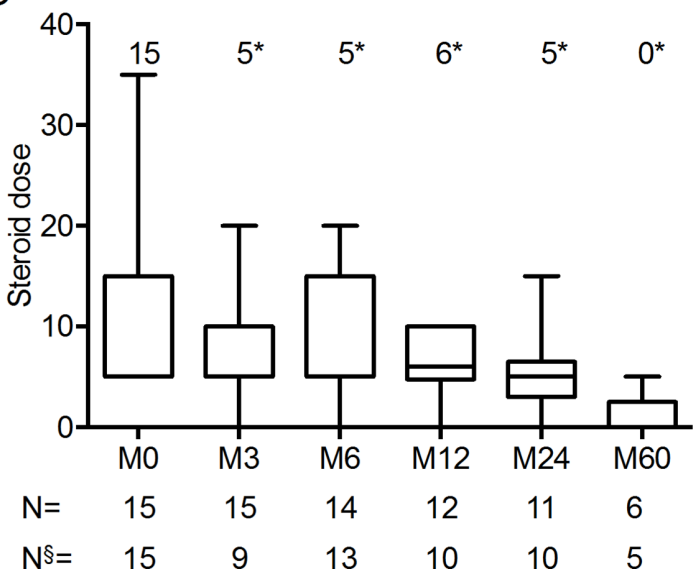

$\mathrm{B}$

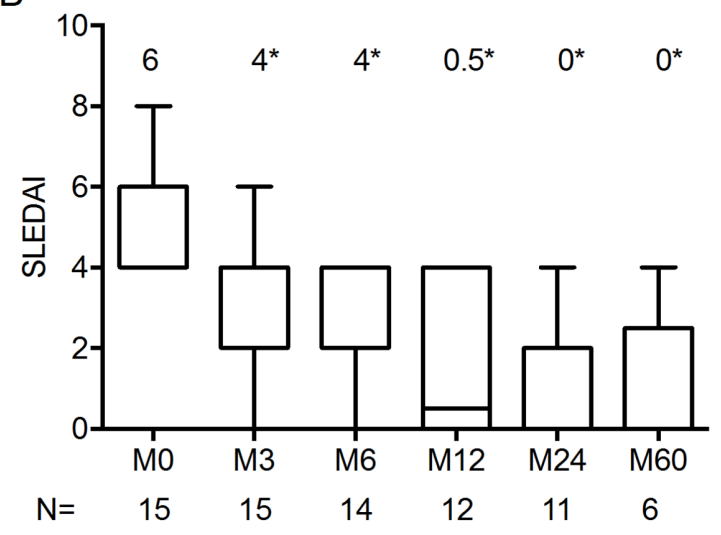

D

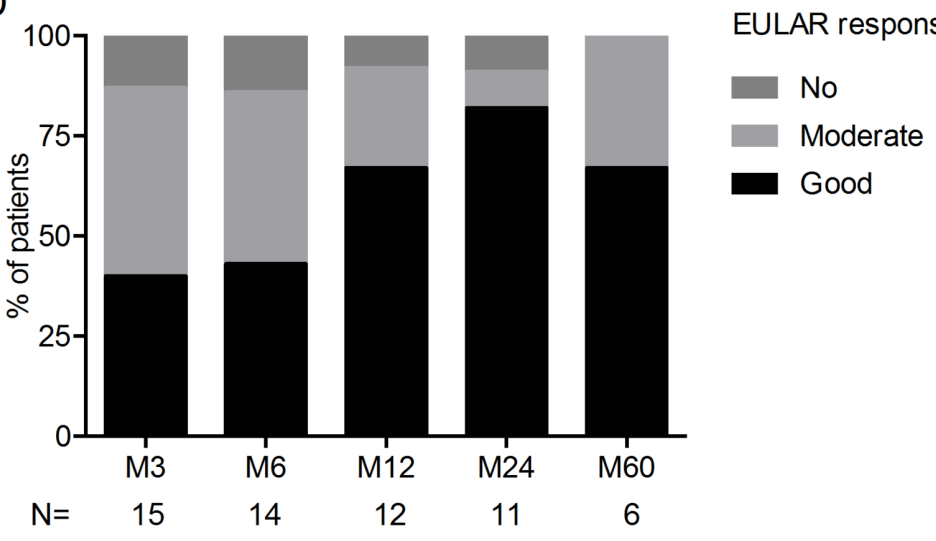

Figure 2 Changes over time in DAS 28 (A), SLEDAI (B), glucocorticoid dose (C) and EULAR response (D) in patients with rhupus treated by anti-TNF- $\alpha$. Box plot (median, quartile, minimum and maximum). Median value is indicated above the box plot. *Significant result (Prob $>97.5 \%$ ) in Bayesian method, compared with baseline (M0). anti-TNF- $\alpha$, antitumour necrosis factor alpha; DAS 28, Disease Activity Score 28; EULAR, European League Against Rheumatism; M, month; n, number of patients. $\mathrm{N}^{\$}$, number of available data; SLEDAI, Systemic Lupus Erythematosus Disease Activity Index.

Arthritis Monitoring and 5.3 per 100 patient-years in the British cohort. ${ }^{30} 31$

Among the limitations of the study are its limited sample size and its non-blinded design due to the low prevalence of rhupus disease. ${ }^{32}$ Rare adverse events may not have been identified in this small population and severe infections hospitalised in other centres may not have been captured. However the sample size is larger than the other open-label studies on rhupus. ${ }^{16-19} 21$ The characteristics of our group of patients with rhupus are similar to those of a cohort of 56 patients with rhupus from China in terms of demographic characteristics and articular involvement. ${ }^{33}$ Extra-articular involvements were different with more mucocutaneous symptoms, less renal involvement and less positivity for extractable nuclear antigens, which may explain the absence of renal or systemic flare in our study. Our data are limited to patients with rhupus in which arthritis is the most prominent component and cannot be generalised to patients with acute renal or cerebral involvements. The long follow-up provides original data regarding the efficacy and tolerance of anti-TNF- $\alpha$ in this disease. No control group was available in our study, but the efficacy of anti-TNF- $\alpha$ was similar to rituximab and abatacept in decreasing DAS 28 in five open-label studies (table 2). DAS 28 was decreased from 5.7 to 3.0 at 6 months in a monocentric study of nine patients with rhupus in Mexico, ${ }^{16}$ from 5.2 to 3.4 at 6 months in five patients with rhupus in Portugal, ${ }^{17}$ from 6.0 to 4.0 at 6 months in six patients in Italy and from 4.9 to 1.6 in three patients. ${ }^{18} 19$ Abatacept was used in six Japanese patients with rhupus in whom the Clinical Disease Activity Index significantly decreased between baseline and 12 weeks, and all patients achieved a good or moderate response according to the EULAR response criteria. ${ }^{21}$ The DAS 28 decreased from 6.2 to 3.6 in three other patients treated by abatacept. ${ }^{19}$ Regarding tolerance with rituximab, one severe and nine non-serious infectious events have occurred in six patients in the first study in 24 months' follow-up, ${ }^{16}$ and other adverse events are related to perfusion. In our study, adverse events were recorded during the 5-year follow-up and cardiovascular events occurred in high-risk patients with several comorbidities. We acknowledge that due to the limited sample size, our findings may not be fully generalised to any patients with rhupus. 


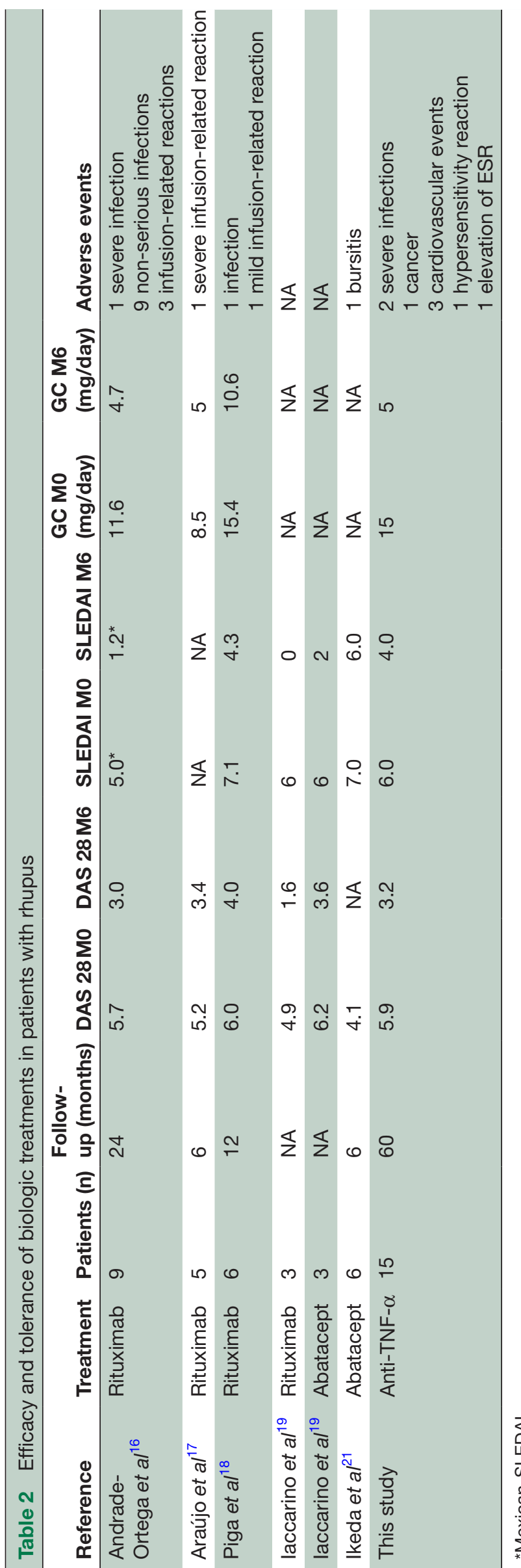

Belimumab, a human monoclonal antibody against B lymphocyte stimulator, was approved by the Food and Drug Administration as an SLE treatment. ${ }^{34}$ The pooled analysis of the two phase III trials of belimumab showed the best results for patients with musculoskeletal and mucocutaneous involvement and may therefore be interesting in rhupus. ${ }^{35}$ Iaccarino $e t a l^{36}$ showed in 64 patients with SLE treated with belimumab that patients with polyarthritis had a significant decrease of DAS 28, but this improvement was due to patients with classical lupus polyarthritis and not in those with rheumatoid-like polyarthritis. Belimumab therefore needs to be evaluated in rhupus.

\section{CONCLUSION}

Overall, our open-label pilot study suggests that antiTNF- $\alpha$ treatment has a favourable efficacy, a decrease in the median dose of glucocorticoids and an acceptable tolerance profile in patients with rhupus with refractive arthritis. Therefore, anti-TNF- $\alpha$ treatments could be an alternative strategy to rituximab in rhupus cases in which arthritis is the most prominent component. Further longterm evaluation and comparative studies are still needed to confirm the favourable benefit/risk ratio of anti-TNF- $\alpha$ therapy in rhupus.

\section{Author affiliations}

${ }^{1}$ Department of Rheumatology, Centre de référence des maladies rares et auto immunes, Hôpitaux Universitaires de Strasbourg, Université de Strasbourg, Strasbourg, France

${ }^{2}$ Fédération de Médecine Translationnelle, Université de Strasbourg, Strasbourg, France

${ }^{3}$ Department of Rheumatology, Centre de compétence maladies rares et autoimmunes, Centre Hospitalier de Mulhouse, Mulhouse, France

${ }^{4}$ UMR 1109 Immuno-Rhumatologie Moléculaire, INSERM, Strasbourg, France

${ }^{5}$ Department of Public Health, Hopitaux universitaires de Strasbourg, Université de Strasbourg, Strasbourg, France

${ }^{6}$ UPR 3572 Immunopathology and Therapeutic Chemistry, CNRS, Strasbourg, France

${ }^{7}$ Department of Internal Medicine, Centre de références des maladies rares et auto immunes, Hopitaux universitaires de Strasbourg, Université de Strasbourg, Strasbourg, France

${ }^{8}$ Department of Rheumatology, Lapeyronie Hospital, Montpellier University, Montpellier, France

${ }^{9}$ Department of Radiology, Hopitaux universitaires de Strasbourg, Université de Strasbourg, Strasbourg, France

${ }^{10}$ Department of Rheumatology, Hopital Louis Pasteur, Colmar, Alsace, France ${ }^{11}$ Department of Internal Medicine, Hopital Louis Pasteur, Colmar, Alsace, France ${ }^{12}$ Department of Immunology, Hopitaux universitaires de Strasbourg, Strasbourg, France

Acknowledgements The authors would like to thank M Breitel (rheumatologist, Strasbourg - France).

Competing interests FD reports personal fees from Gilead outside the submitted work. LS reports personal fees from AbbVie, Pfizer, Roche and Roche Chugai, outside the submitted work. LA reports consulting fees from Amgen, GSK, Lilly and AstraZeneca. TM reports personal fees from GSK and Merck, during the conduct of the study. J-EG reports research grants and consulting fees from AbbVie, BMS, MSD, UCB, Pfizer and Roche. JS reports personal fees from AbbVie, BMS, MSD, UCB, Pfizer, Roche, Novartis, GSK, Actelion, Amgen and Hospira, during the conduct of the study, and consultancies and speaking fees from AbbVie, Roche, Pfizer, Merck, UCB, Novartis, BMS and Actelion. CS reports personal fees from AbbVie and Pfizer, outside the submitted work. 
Ethics approval The study protocol was approved by the Clinical Research Ethics Committee of Strasbourg University Hospital.

Provenance and peer review Not commissioned; externally peer reviewed.

Open Access This is an Open Access article distributed in accordance with the Creative Commons Attribution Non Commercial (CC BY-NC 4.0) license, which permits others to distribute, remix, adapt, build upon this work non-commercially, and license their derivative works on different terms, provided the original work is properly cited and the use is non-commercial. See: http://creativecommons.org/ licenses/by-nc/4.0/

C) Article author(s) (or their employer(s) unless otherwise stated in the text of the article) 2017. All rights reserved. No commercial use is permitted unless otherwise expressly granted.

\section{REFERENCES}

1. Schur P. Systemic lupus erythematosus. In: Beeson PB, Mc Dermott W, eds. Cecil-loeb textbook of medicine. Philadelphia, PA: WB Saunders Company, 1971:821.

2. Elliott MJ, Maini RN, Feldmann M, et al. Randomised double-blind comparison of chimeric monoclonal antibody to tumour necrosis factor alpha (cA2) versus placebo in rheumatoid arthritis. Lancet 1994:344:1105-10.

3. Eriksson C, Engstrand S, Sundqvist KG, et al. Autoantibody formation in patients with rheumatoid arthritis treated with anti-TNF alpha. Ann Rheum Dis 2005;64:403-7.

4. De Rycke L, Kruithof E, Van Damme N, et al. Antinuclear antibodies following infliximab treatment in patients with rheumatoid arthritis or spondylarthropathy. Arthritis Rheum 2003;48:1015-23.

5. De Bandt M, Sibilia J, Le Loët X, et al. Systemic lupus erythematosus induced by anti-tumour necrosis factor alpha therapy: a French national survey. Arthritis Res Ther 2005;7:R545-51.

6. Gordon C, Ranges GE, Greenspan JS, et al. Chronic therapy with recombinant tumor necrosis factor-alpha in autoimmune NZB/NZW F1 mice. Clin Immunol Immunopathol 1989;52:421-34.

7. Jacob $\mathrm{CO}, \mathrm{McDevitt} \mathrm{HO}$. Tumour necrosis factor-alpha in murine autoimmune 'lupus' nephritis. Nature 1988;331:356-8.

8. Aringer M, Smolen JS. Cytokine expression in lupus kidneys. Lupus 2005;14:13-18.

9. Zampieri S, Alaibac M, laccarino L, et al. Tumour necrosis factor alpha is expressed in refractory skin lesions from patients with subacute cutaneous lupus erythematosus. Ann Rheum Dis 2006;65:545-8.

10. Gerl V, Hostmann B, Johnen C, et al. The intracellular 52-kd Ro/ SSA autoantigen in keratinocytes is up-regulated by tumor necrosis factor alpha via tumor necrosis factor receptor I. Arthritis Rheum 2005;52:531-8.

11. Aringer $M$, Graninger WB, Steiner $G$, et al. Safety and efficacy of tumor necrosis factor alpha blockade in systemic lupus erythematosus: an open-label study. Arthritis Rheum 2004;50:3161-9.

12. Aringer M, Steiner G, Graninger WB, et al. Effects of shortterm infliximab therapy on autoantibodies in systemic lupus erythematosus. Arthritis Rheum 2007;56:274-9.

13. Aringer M, Houssiau F, Gordon C, et al. Adverse events and efficacy of TNF-alpha blockade with infliximab in patients with systemic lupus erythematosus: long-term follow-up of 13 patients. Rheumatology 2009;48:1451-4.

14. Uppal SS, Hayat SJ, Raghupathy R. Efficacy and safety of infliximab in active SLE: a pilot study. Lupus 2009;18:690-7.

15. Cortés-Hernández J, Egri N, Vilardell-Tarrés M, et al. Etanercept in refractory lupus arthritis: an observational study. Semin Arthritis Rheum 2015;44:672-9.

16. Andrade-Ortega L, Irazoque-Palazuelos F, Muñóz-López S, et al. Efficacy and tolerability of rituximab in patients with rhupus. Reumatol Clin 2013;9:201-5.

17. Araújo F, Silva I, Sepriano A, et al. Off-label use of rituximab in systemic rheumatic diseases: case series and review. Acta Reumatol Port 2013;38:290-4.
18. Piga M, Gabba A, Cauli A, et al. Rituximab treatment for 'rhupus syndrome': clinical and power-Doppler ultrasonographic monitoring of response. a longitudinal pilot study. Lupus 2013;22:624-8.

19. Iaccarino L, Gatto M, Bettio S, et al. Overlap connective tissue disease syndromes. Autoimmun Rev 2013;12:363-73.

20. Cobo-Ibáñez T, Loza-Santamaría E, Pego-Reigosa JM, et al. Efficacy and safety of rituximab in the treatment of non-renal systemic lupus erythematosus: a systematic review. Semin Arthritis Rheum 2014;44:175-85.

21. Ikeda K, Sanayama Y, Makita S, et al. Efficacy of abatacept for arthritis in patients with an overlap syndrome between rheumatoid arthritis and systemic lupus erythematosus. Clin Dev Immunol 2013;2013:1-10.

22. Merrill JT, Burgos-Vargas R, Westhovens R, et al. The efficacy and safety of abatacept in patients with non-life-threatening manifestations of systemic lupus erythematosus: results of a twelvemonth, multicenter, exploratory, phase Illb, randomized, doubleblind, placebo-controlled trial. Arthritis Rheum 2010;62:3077-87.

23. Arnett FC, Edworthy SM, Bloch DA, et al. The American rheumatism association 1987 revised criteria for the classification of rheumatoid arthritis. Arthritis Rheum 1988:31:315-24.

24. Hochberg MC. Updating the American College of Rheumatology revised criteria for the classification of systemic lupus erythematosus. Arthritis Rheum 1997;40:1725.

25. Petri M, Kim MY, Kalunian KC, et al. Combined oral contraceptives in women with systemic lupus erythematosus. N Engl J Med 2005;353:2550-8.

26. Wells G, Becker JC, Teng J, et al. Validation of the 28-joint Disease Activity Score (DAS28) and European league against rheumatism response criteria based on c-reactive protein against disease progression in patients with rheumatoid arthritis, and comparison with the DAS28 based on erythrocyte sedimentation rate. Ann Rheum Dis 2009;68:954-60.

27. van Gestel AM, Haagsma CJ, van Riel PL. Validation of rheumatoid arthritis improvement criteria that include simplified joint counts. Arthritis Rheum 1998;41:1845-50.

28. Wollaston SJ, Farewell VT, Isenberg DA, et al. Defining response in systemic lupus erythematosus: a study by the systemic lupus international collaborating clinics group. J Rheumatol 2004:31:2390-4.

29. Jacob CO, Fronek Z, Lewis GD, et al. Heritable major histocompatibility complex class II-associated differences in production of tumor necrosis factor alpha: relevance to genetic predisposition to systemic lupus erythematosus. Proc Natl Acad Sci U S A 1990;87:1233-7.

30. van Dartel SA, Fransen J, Kievit W, et al. Predictors for the 5-year risk of serious infections in patients with rheumatoid arthritis treated with anti-tumour necrosis factor therapy: a Cohort Study in the Dutch Rheumatoid Arthritis Monitoring (DREAM) registry. Rheumatology 2013:52:1052-7.

31. Dixon WG, Watson K, Lunt M, et al. Rates of serious infection, including site-specific and bacterial intracellular infection, in rheumatoid arthritis patients receiving anti-tumor necrosis factor therapy: results from the british society for rheumatology biologics register. Arthritis Rheum 2006;54:2368-76.

32. Panush RS, Edwards NL, Longley S, et al. 'Rhupus' syndrome. Arch Intern Med 1988;148:1633-6.

33. Li J, Wu H, Huang X, et al. Clinical analysis of 56 patients with rhupus syndrome: manifestations and comparisons with systemic lupus erythematosus: a retrospective case-control study. Medicine 2014;93:e49.

34. Navarra SV, Guzmán RM, Gallacher AE, et al. Efficacy and safety of belimumab in patients with active systemic lupus erythematosus: a randomised, placebo-controlled, phase 3 trial. Lancet 2011;377:721-31.

35. Manzi S, Sánchez-Guerrero J, Merrill JT, et al. Effects of belimumab, a B lymphocyte stimulator-specific inhibitor, on disease activity across multiple organ domains in patients with systemic lupus erythematosus: combined results from two phase III trials. Ann Rheum Dis 2012;71:1833-8.

36. laccarino L, Bettio S, Reggia R, et al. Effects of belimumab on flare rate and expected damage progression in patients with active systemic lupus erythematosus. Arthritis Care Res 2017;69:115-23. 